\title{
Polyunsaturated Phospholipid Modified Membrane Degradation Catalyzed by a Secreted Phospholipase A2 - Supporting Information
}

Pin Zhang, ${ }^{1}$ Veronica Villanueva, ${ }^{1}$ Joseph Kalkowski, ${ }^{1}$ Chang Liu, ${ }^{1}$ Tiep Pham, ${ }^{1}$ Ursula Perez-Salas, ${ }^{2}$ Wei Bu, ${ }^{3}$ Binhua Lin, ${ }^{3}$ Ying Liu ${ }^{l} *$

${ }^{1}$ Department of Chemical Engineering, University of Illinois at Chicago, Chicago, Illinois 60607;

${ }^{2}$ Department of Physics, University of Illinois at Chicago, Chicago, Illinois 60607;

${ }^{3}$ NSF's ChemMatCARS, University of Chicago, Illinois 60637;

S1. Molecular structures and degradation catalyzed by $\mathrm{SPLA}_{2}$

A DPPC 1-palmitoyl-2-hydroxy-sn-glycero-3-phosphocholine

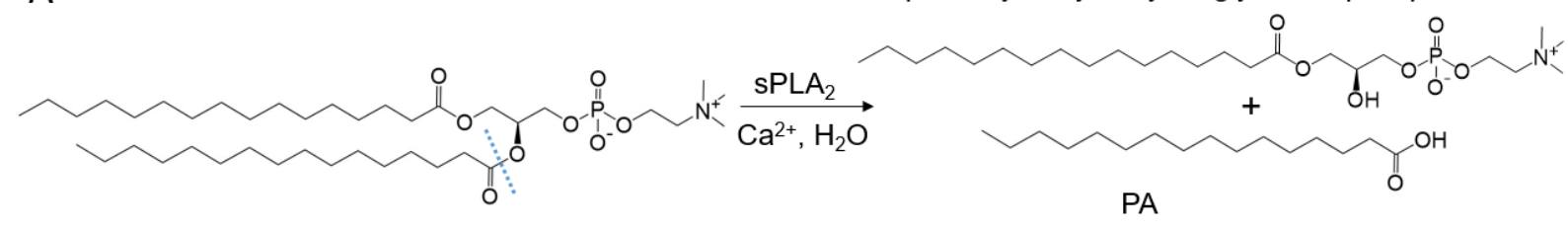

B DLPC

1-linoleoyl-2-hydroxy-sn-glycero-3-phosphocholine
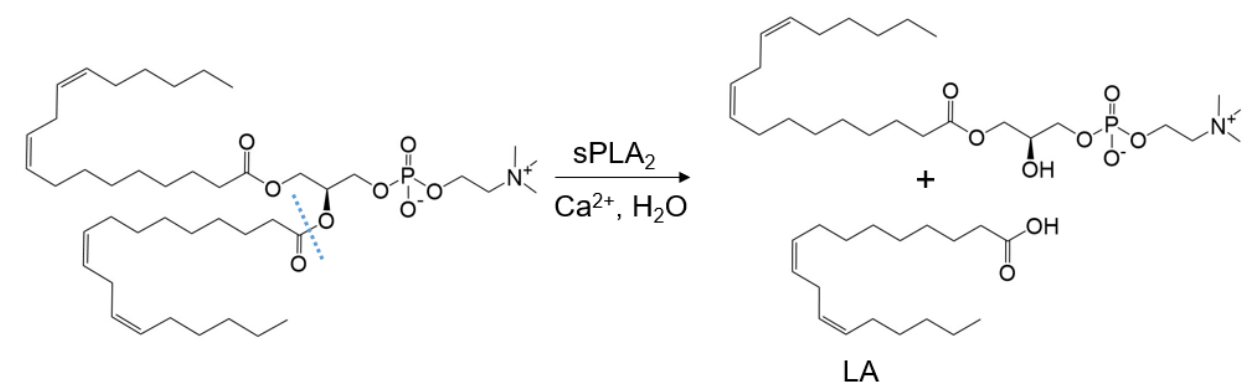

Figure S1. Molecular structures and $\mathrm{SPLA}_{2}$-catalyzed degradation of (A) DPPC and (B) DLPC.

S2. Modified electroformation method

The sandwich chamber contained two ITO-coated glass slides (Figure S2 A). The conductive side of the top glass slide was spin-coated with lipid film and faced toward the conductive side of the bottom glass slide, which was glued to a piece of copper tape using a silver-filled adhesive (AI Technology, Inc.). Electrical tape was used to further fix the position of the copper tape on the bottom glass slide. The two glass slides, spaced by an O-ring (ID 0.614 in and OD 0.754 in, 
McMaster-Carr), were fastened together using two clamps covered with electrical tapes. The assembled chamber was filled with $50^{\circ} \mathrm{C} 0.5 \mathrm{mM}$ Tris buffer. The setup was placed in a $50^{\circ} \mathrm{C}$ oven and connected by two electricity conducting clamps to a $1 \mathrm{~V} 10 \mathrm{~Hz}$ AC field for 1.5 hours. After electroformation, GUVs were collected by using a glass pipette from the sandwich chamber. Typical DPPC GUVs and DPPC-DLPC GUVs generated using this method are displayed in Figure S2 B and C, respectively.
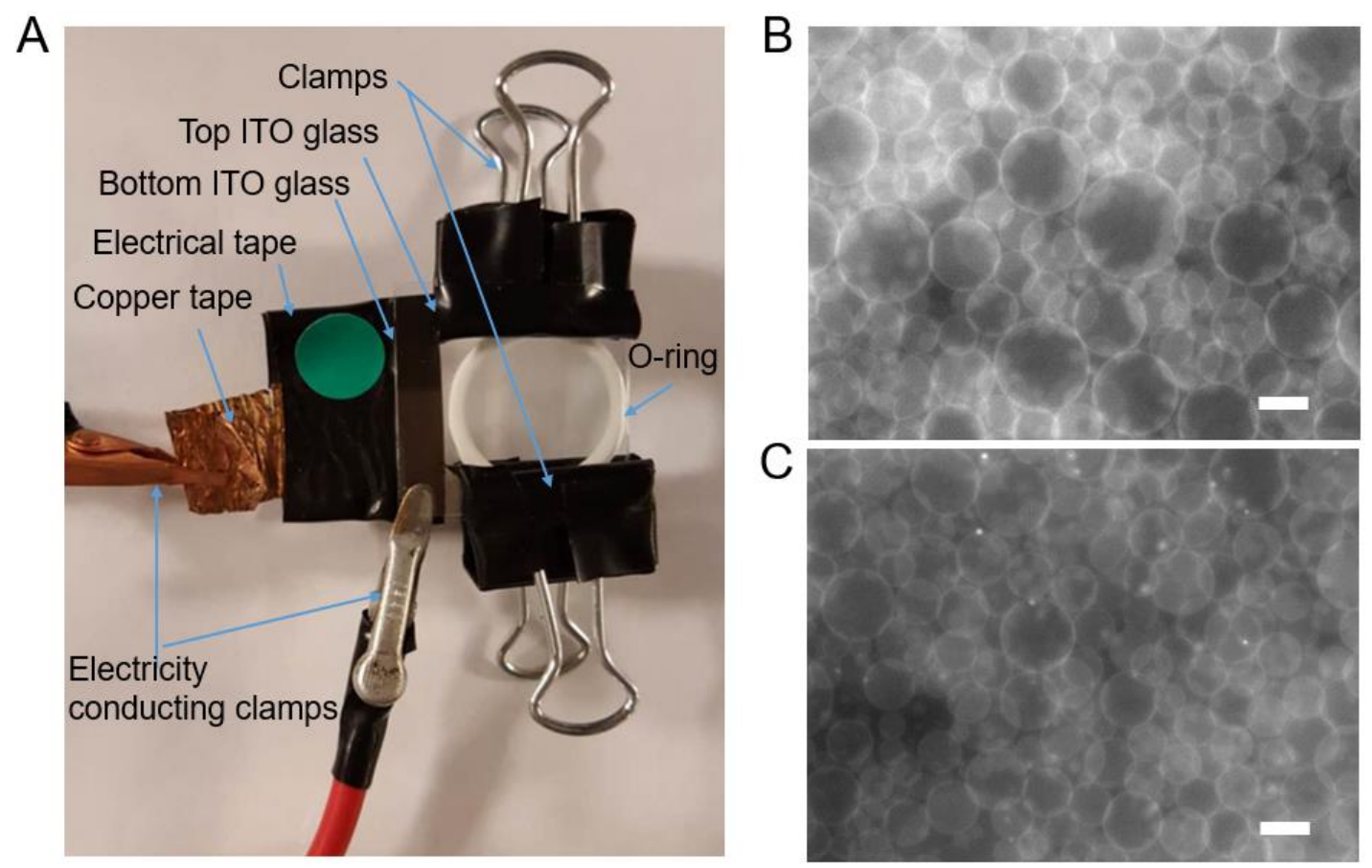

Figures S2. GUVs were prepared through modified electroformation method. (A) Setup of the sandwich chamber. (B) Images of DPPC GUVs and (C) DPPC-DLPC GUVs with DLPC fraction $\mathrm{x}_{\mathrm{DLPC}}=0.63$ after electroformation. The scale bars in all frames are $20 \mu \mathrm{m}$.

S3. Observations of GUVs without $\mathrm{SPLA}_{2}$

Control experiments were conducted for DPPC and DPPC-DLPC GUVs using a buffer containing the same $\mathrm{Ca}^{2+}$ concentration $(150 \mu \mathrm{M})$ but no enzyme. For the DPPC GUVs, no obvious phase separation was observed on the vesicle surface before or after mixing with the buffer (Figure S3A). For the DPPC-DLPC GUVs, small and irregularly shaped domains were observed on the surface of some GUVs (Figure S3B), and these domains remained the same 
throughout the control experiments (Figure S3C). Both types of GUVs were stable during the experimental measurements, and no size or morphology change was observed.
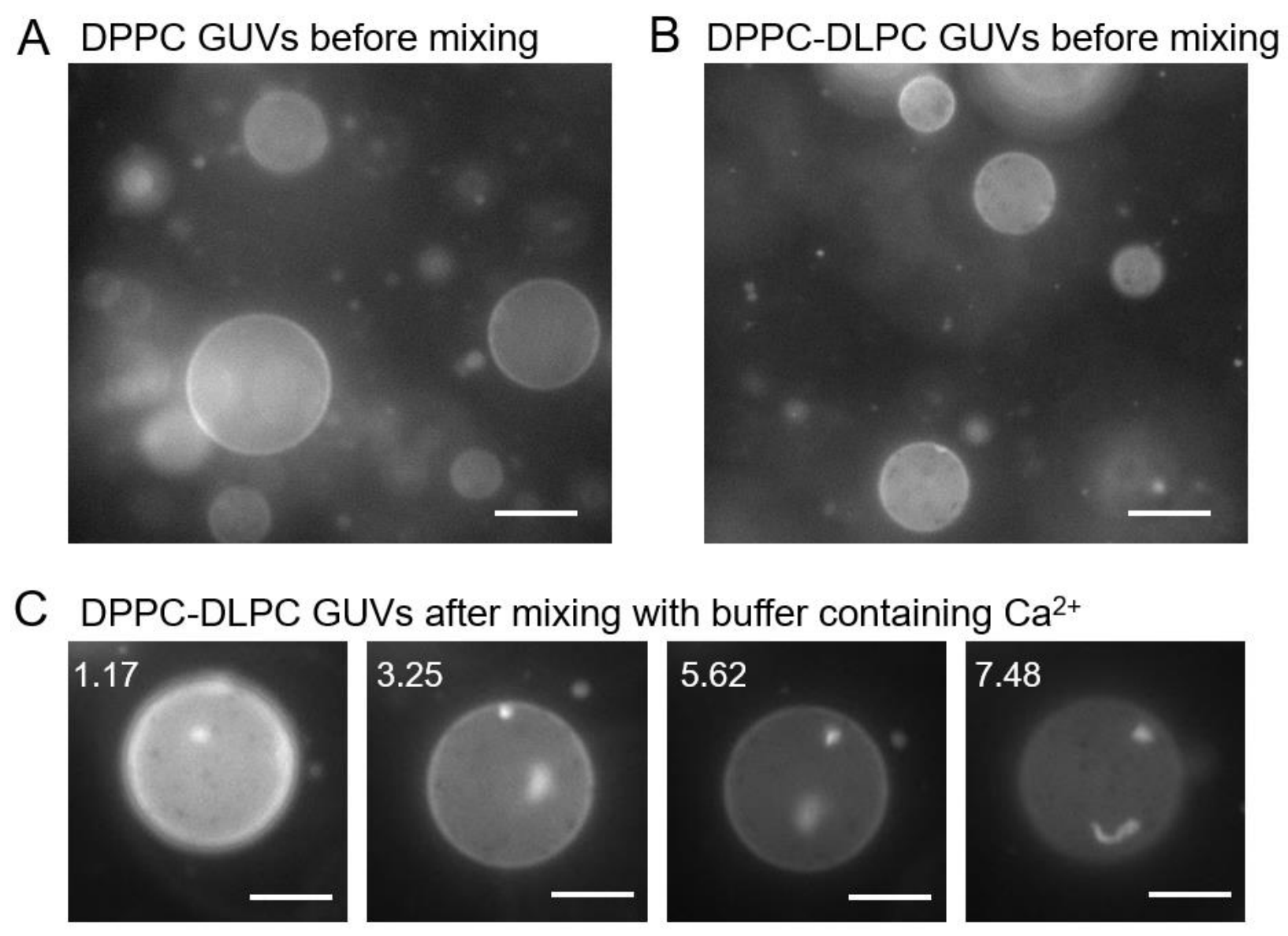

Figure S3. Images of DPPC and DPPC-DLPC GUVs. (A) No phase separation was observed on DPPC GUVs before mixing with a buffer containing $150 \mu \mathrm{M} \mathrm{Ca}^{2+}$. (B) Small domains appeared on the surface of some DPPC-DLPC GUVs before mixing with a buffer containing 150 $\mu \mathrm{M} \mathrm{Ca}^{2+}$. (C) Domains remained on the surface of DPPC-DLPC GUVs throughout the control experiments upon addition of the buffer. The time (in minutes) after mixing with the buffer is displayed in the upper left corner of each frame in C. The scale bar in each frame represents 20 $\mu \mathrm{m}$. Exposure times were auto-adjusted to provide the best contrast. 
S4. Exposure time of the fluorescence images

Table S1. Exposure time corresponding to time-lapse images of DPPC GUVs and DPPC-DLPC GUVs with DLPC fraction $x_{D L P C}=0.63$ after mixture with $\mathrm{SPLA}_{2}$ at $23^{\circ} \mathrm{C}$. Exposure time (milliseconds) are listed according to the order of the frames presented in Figure 1A and 1B.

\begin{tabular}{|c|c|c|c|c|c|c|c|}
\hline GUVs & $\begin{array}{c}\text { sPLA }_{2} \text { conc. } \\
(\mu \mathrm{g} / \mathrm{mL})\end{array}$ & Frame 1 & Frame 2 & Frame 3 & Frame 4 & Frame 5 & Frame 6 \\
\hline \multirow{2}{*}{ DPPC } & 0.1 & 110 & 110 & 126 & 112 & 141 & 156 \\
\cline { 2 - 8 } & 5 & 469 & 320 & 338 & 304 & 229 & 725 \\
\hline \multirow{2}{*}{$\begin{array}{c}\text { DPPC- } \\
\text { DLPC }\end{array}$} & 0.1 & 821 & 804 & 811 & 800 & 826 & 774 \\
\cline { 2 - 8 } & 5 & 287 & 511 & 511 & 326 & 443 & 524 \\
\hline
\end{tabular}

S5. Lateral packing structural parameters of lipid films before and after degradation

Table S2. Lateral packing structural parameters of DPPC and DPPC-DLPC monolayers at gasliquid interface resulted from GIXD measurements.

\begin{tabular}{|c|c|c|c|}
\hline Parameter & $\mathrm{x}_{\mathrm{DLPC}}=0$ & $\mathrm{x}_{\mathrm{DLPC}}=0.2$ & $\mathrm{x}_{\mathrm{DLPC}}=0.4$ \\
\hline $\mathrm{Q}_{\mathrm{xy}(1,1)}\left(\AA^{-1}\right)$ & $1.33^{ \pm 0.004}$ & $1.334^{ \pm 0.003}$ & $1.334^{ \pm 0.003}$ \\
\hline $\mathrm{Q}_{\mathrm{xy}(0,2)}\left(\AA^{-1}\right)$ & $1.462^{ \pm 0.001}$ & $1.462^{ \pm 0.001}$ & $1.465^{ \pm 0.001}$ \\
\hline $\mathrm{Q}_{\mathrm{z}(1,1)}\left(\AA^{-1}\right)$ & $0.745^{ \pm 0.007}$ & $0.736^{ \pm 0.013}$ & $0.760^{ \pm 0.046}$ \\
\hline $\mathrm{a}(\AA)$ & $5.7^{ \pm 0.1}$ & $5.6^{ \pm 0.1}$ & $5.6^{ \pm 0.1}$ \\
\hline $\mathrm{b}(\AA)$ & $8.6^{ \pm 0.1}$ & $8.6^{ \pm 0.1}$ & $8.6^{ \pm 0.1}$ \\
\hline Tilt angle $\left(^{\circ}\right)$ & $33.9^{ \pm 0.5}$ & $33.4^{ \pm 0.6}$ & $34.3^{ \pm 1.8}$ \\
\hline Unit area per tail $\left(\AA^{2}\right)$ & $24.3^{ \pm 0.1}$ & $24.2^{ \pm 0.1}$ & $24.1^{ \pm 0.1}$ \\
\hline
\end{tabular}


Table S3. GIXD peak positions corresponding to five GIXD Bragg peaks of PA film, degraded DPPC film, and degraded DPPC-DLPC film ( $\mathrm{x}_{\mathrm{DLPC}}=0.2$ and 0.4$)$ at gas-liquid interface. Peak positions $\left(\AA^{-1}\right)$ are listed according to the order of the peaks labeled in Figure $4 \mathrm{~A}$.

\begin{tabular}{|c|c|c|c|c|}
\hline Peak No. & PA & $\begin{array}{c}\text { Degraded } \\
\text { DPPC }\end{array}$ & $\begin{array}{c}\text { Degraded } \\
\text { DPPC-DLPC } \\
\left(\mathrm{x}_{\text {DLPC }}=0.2\right)\end{array}$ & $\begin{array}{c}\text { Degraded } \\
\text { DPPC-DLPC } \\
\left(\mathrm{x}_{\text {DLPC }}=0.4\right)\end{array}$ \\
\hline 1 & $1.074^{ \pm 0.003}$ & $1.076^{ \pm 0.005}$ & $1.075^{ \pm 0.002}$ & $1.075^{ \pm 0.030}$ \\
\hline 2 & $1.417^{ \pm 0.001}$ & $1.414^{ \pm 0.001}$ & $1.413^{ \pm 0.001}$ & $1.412^{ \pm 0.001}$ \\
\hline 3 & $1.526^{ \pm 0.001}$ & $1.527^{ \pm 0.001}$ & $1.529^{ \pm 0.001}$ & $1.537^{ \pm 0.003}$ \\
\hline 4 & $1.619^{ \pm 0.001}$ & $1.611^{ \pm 0.001}$ & $1.609^{ \pm 0.001}$ & $1.609^{ \pm 0.002}$ \\
\hline 5 & $1.845^{ \pm 0.001}$ & $1.843^{ \pm 0.001}$ & $1.843^{ \pm 0.001}$ & $1.843^{ \pm 0.002}$ \\
\hline
\end{tabular}

